\title{
The current state of drug repurposing and rare diseases: an interview with Paul Trippier
}

\author{
Paul C Trippier*,1 \\ ${ }^{1}$ Department of Pharmaceutical Sciences, University of Nebraska Medical Center, NE 68198, USA \\ *Author for correspondence: paul.trippier@unmc.edu
}

Paul Tripper is an Associate Professor of Medicinal Chemistry at the University of Nebraska Medical Center (UNMC, NE, USA) and an Editorial Board member of Future Drug Discovery. Here, he speaks to Managing Editor Francesca Lake about drug repurposing, focusing on the key challenges, its application to rare diseases and what we can look forward to in the future.

First draft submitted: 12 December 2019; Accepted for publication: 21 January 2020; Published online: 13 March 2020

Keywords: ALS • drug repurposing • IPSC models • PROTACS • rare diseases

\section{Please introduce yourself $\&$ tell us about your career to date $\&$ your current research}

I am an Associate Professor of Pharmaceutical Sciences at the University of Nebraska Medical Center (UNMC) in the College of Pharmacy and the UNMC Center for Drug Discovery and a Member of the Fred \& Pamela Buffett Cancer Center (NE, USA). I received a MChem degree in Chemistry, Toxicology and Drug Discovery from the University of Hull (Hull, UK) and a D.Phil. from Merton College Oxford (Oxford, UK), working on the total synthesis of a bioactive natural product with Mark Moloney. I then undertook postdoctoral research focusing on medicinal chemistry and chemical biology with Chris McGuigan at Cardiff University (UK) and with Richard Silverman at Northwestern University (IL, USA). I stayed in the USA to set up my independent research group at Texas Tech University Health Sciences Center (TX, USA) as an Assistant Professor. In 2019, I was promoted to Associate Professor and in July my group moved to UNMC. My research is focused on early stage small molecule drug discovery, applying synthetic organic chemistry to the discovery of new bioactive compounds and optimizing these compounds as experimental therapeutics. We have particular interests in cancer and neurodegenerative disease drug discovery, but we do work on any area of unmet medical need. Our current research is directed toward developing treatment options for a rare pediatric and other neurodegenerative diseases, a number of cancers and stroke.

\section{What is the current state of the drug repurposing field: what recent advances $\&$ successes} have you found exciting?

I think the field is enjoying its time in the limelight [1]. The rise to prominence of phenotypic screening models over the past few years, especially from induced pluripotent stem cells (iPSCs) obtained directly from patients, has allowed the field to rapidly screen clinically approved compounds for beneficial effects in high-quality models of disease. Such screening relies on phenotype, not artificial overexpression or knock out of a particular gene or protein, allowing for rapid identification of initial beneficial effect that is not reliant on a particular target [2].

The most exciting advance in the area recently has not really been a drug repurposed to treat a different disease, the standard definition of drug repurposing, but a drug repurposed to introduce a new mechanism to design drugs; degradation. The field of proteolysis targeting chimeras (PROTACS) has been rising rapidly, based on the idea of targeting proteins for degradation instead of inhibiting them [3]. Thalidomide-based cereblon ligands have become one of the major classes of E3 ubiquitin ligase recruiting motifs used in PROTAC design [4]. This field offers tremendous potential to develop drugs for targets that have previously been seen as 'undruggable' [5]. 
Related to my past and continuing research in amyotrophic lateral sclerosis (ALS) [6,7], I was closely following the clinical trial of the now discontinued anticonvulsant retigabine [8], in ALS patients (NCT02450552). To date it has been shown to lower motor neuron excitability in patients and has further promise. The Phase II trial of mycophenolate mofetil for CLN3 Disease (NCT01399047) was a rollercoaster of feelings; excitement that a trial featuring a repurposed drug for this particular rare disease that we work on [9], was instigated, but disappointment at the news that it was deemed safe but clinically ineffective, at least in short-term studies [10]. Longer-term studies are warranted.

\section{What challenges do we still face? How might we overcome them?}

Data curating and access is a challenge; much patient data on performance of a drug is locked behind firewalls, which is absolutely necessary to protect the patient. However, deidentified data could be tremendously useful for analyzing new opportunities for repurposing. What may be noted as a curious side effect in one indication could be a useful treatment modality in another indication. The story of Viagra developed initially as an antihypertensive comes to mind.

In basic research, the NIH clinical collection libraries are an excellent resource for screening clinically approved drugs for repurposing opportunities but making the results of these screens publicly available would be of great help. Likewise, publishing negative results, that certain drugs were tried but failed to elicit a response, would save valuable time and resources. Perhaps the area of big data can be applied to drug repurposing [11].

What's more, perhaps expanding the definition of repurposing a little would be a good idea; compounds that have reached late-stage clinical trial but have not yet received approval could be screened for repurposing. This would potentially allow accelerated repurposing efforts, although this would need to be in close collaboration with the company holding the intellectual property.

\section{How is drug repurposing being applied in the tricky field of rare disease?}

With the advent of patient-derived iPSC models and their increasing availability for more rare diseases, drug repurposing is being applied to more and more rare diseases. It is certainly possible that compounds that have already been developed for other indications could be of benefit to rare diseases [12]. Given the enormous costs of developing new drugs, repurposing for rare diseases, whereby the very definition is that not many patients will be taking the drug, offers financial incentives for pharmaceutical companies to screen clinical compounds about to come off patent for new indications [13]. Repurposing represents a much more rapid route to clinical trials over a new compound; all of the safety and interaction studies have already been done. This allows patients to benefit faster.

\section{What more would you like to see done in this arena?}

The clinically approved compounds are there and relatively easily accessible for research groups. The need at the moment and that has been a need for several years in drug discovery for rare diseases, is better models of disease phenotypes and better models of cell systems in a dish. Patient-derived iPSC-derived neurons are providing excellent phenotypic screening platforms for rare neurodegenerative diseases but many rare diseases do not have such models available. A coordinated effort by industry, academia and the government to develop and make freely available at least one iPSC model of each rare disease would be a tremendous service to the field of drug discovery, as the human genome project was.

Equally, the identification of suitable biomarkers to be able to track patient outcomes in clinical trials is urgently needed for many rare diseases [14]. How do we track that the drug is actually working? This is a particularly problematic area as we still lack biomarkers for several common diseases let alone rare diseases. Finally, more funding for repurposing efforts in rare diseases underline these preceding points. The National Clinical Assessment and Treatment Service at the NIH has done a tremendous job in providing greater resources for rare diseases but more funding would provide even more advances, which may be applicable outside the rare disease in question. Perhaps one day, a drug approved for a rare disease can be repurposed to treat a more common indication.

\section{If you had access to unlimited funding, what would be the first thing you would like to do in terms of drug repurposing \& rare diseases?}

I will run with my suggestion from above. Create a public repository for rare disease patient-derived iPSCs and make these cells available to any researcher for free. We would also offer high-throughput screening facilities to 
identify drugs suitable for repurposing. As we have unlimited funding, we would also offer our services as medicinal chemists to use these drugs as hit compounds for optimization of more potent drug candidates. This is an area a little outside the classical definition of repurposing, but one that is equally important in my mind. Rare diseases generally lack any chemical scaffold that has a positive effect to rescue phenotypes; even identification of a low potency compound can be the starting point for a medicinal chemistry optimization campaign [15]. Repurposing does not need to start and end with the clinically approved drug, repurposing can provide a hit compound for further development.

\section{Financial \& competing interests disclosure}

P Trippier is an advisor to Circumvent Pharmaceuticals and is a named inventor on the following patent describing drug repurposing for neurodegenerative disease: functionalized pyridine carbamates with enhanced neuroprotective activity. PCT Int. Appl. (2019), WO2019014547A8 \& 20190117. P Trippier's research is currently funded by NIH grants: R01 CA226436 and R01NS106879. The author has no other relevant affiliations or financial involvement with any organization or entity with a financial interest in or financial conflict with the subject matter or materials discussed in the manuscript apart from those disclosed.

No writing assistance was utilized in the production of this manuscript.

\section{Interview disclosure}

The opinions expressed in this interview are those of $\mathrm{P}$ Trippier and do not necessarily represent the official views of the National Institutes of Health or reflect the views of Future Science Ltd.

\section{Open access}

This work is licensed under the Attribution-NonCommercial-NoDerivatives 4.0 Unported License. To view a copy of this license, visit http://creativecommons.org/licenses/by-nc-nd/4.0/

\section{References}

1. Pushpakom S, Iorio F, Eyers PA et al. Drug repurposing: progress, challenges and recommendations. Nat. Rev. Drug Discov. 18(1), 41-58 (2019).

2. Rowe RG, Daley GQ. Induced pluripotent stem cells in disease modelling and drug discovery. Nat. Rev. Genet. 20(7), 377-388 (2019).

3. Sakamoto KM, Kim KB, Kumagai A, Mercurio F, Crews CM, Deshaies RJ. PROTACS: chimeric molecules that target proteins to the Skp1-Cullin-F box complex for ubiquitination and degradation. Proc. Natl Acad. Sci. USA 98(15), 8554-8559 (2001).

4. Chopra R, Sadok A, Collins I. A critical evaluation of the approaches to targeted protein degradation for drug discovery. Drug Discov. Today Technol. 31, 5-13 (2019).

5. Lai AC, Crews CM. Induced protein degradation: an emerging drug discovery paradigm. Nat. Rev. Drug Discov. 16(2), 101-114 (2017).

6. Trippier PC, Benmohamed R, Kirsch DR, Silverman RB. Substituted pyrazolones require $\mathrm{N}_{2}$ hydrogen bond donating ability to protect against cytotoxicity from protein aggregation of mutant superoxide dismutase 1. Bioorg. Med. Chem. Lett. 22(21), 6647-6650 (2012).

7. Trippier PC, Zhao KT, Fox SG et al. Proteasome activation is a mechanism for pyrazolone small molecules displaying therapeutic potential in amyotrophic lateral sclerosis. ACS Chem. Neurosci. 5(9), 823-829 (2014).

8. Wainger BJ, Kiskinis E, Mellin C et al. Intrinsic membrane hyperexcitability of amyotrophic lateral sclerosis patient-derived motor neurons. Cell Rep. 7(1), 1-11 (2014).

9. Makoukji J, Saadeh F, Mansour KA et al. Flupirtine derivatives as potential treatment for the neuronal ceroid lipofuscinoses. Ann. Clin. Transl. Neurol. 5(9), 1089-1103 (2018).

10. Augustine EF, Beck CA, Adams HR et al. Short-term administration of mycophenolate is well-tolerated in CLN3 disease (juvenile neuronal ceroid lipofuscinosis). JIMD Rep. 43, 117-124 (2019).

11. Brown N, Cambruzzi J, Cox PJ et al. Big data in drug discovery. Prog. Med. Chem. 57(1), 277-356 (2018).

12. Kinarivala N, Trippier PC. Progress in the development of small molecule therapeutics for the treatment of neuronal ceroid lipofuscinoses (NCLs). J. Med. Chem. 59(10), 4415-4427 (2016).

13. Cha Y, Erez T, Reynolds IJ et al. Drug repurposing from the perspective of pharmaceutical companies. Br. J. Pharmacol. 175(2), 168-180 (2018).

14. Gulbakan B, Ozgul RK, Yuzbasioglu A, Kohl M, Deigner HP, Ozguc M. Discovery of biomarkers in rare diseases: innovative approaches by predictive and personalized medicine. EPMA J. 7(1), 24 (2016).

15. Kinarivala N, Patel R, Boustany RM, Al-Ahmad A, Trippier PC. Discovery of aromatic carbamates that confer neuroprotective activity by enhancing autophagy and inducing the anti-apoptotic protein B-cell lymphoma 2 (Bcl-2). J. Med. Chem. 60(23), 9739-9756 (2017). 